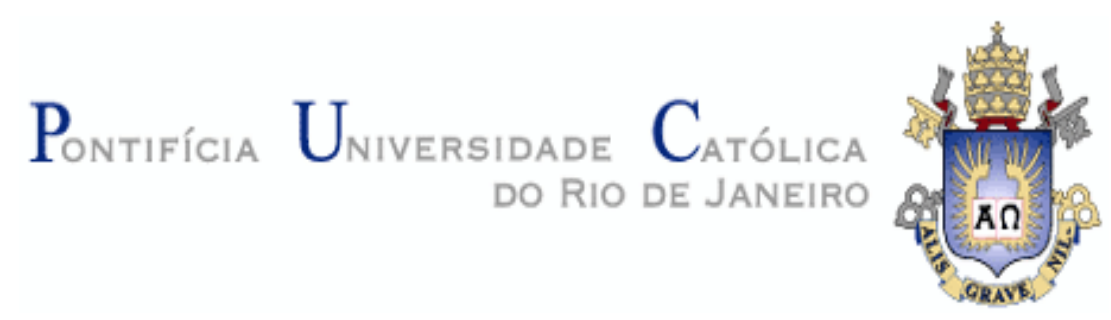

Roberta Amorim de Assis

\begin{abstract}
Aperfeiçoamento e aplicações de uma metodologia para análise de especiação de arsênio por eletroforese capilar com detector de ICPMS
\end{abstract}

Tese de Doutorado

Tese apresentada como requisito parcial para obtenção do título de Doutor pelo Programa de PósGraduação em Química da PUC-Rio.

Orientadores: Prof. Dr. Norbert Miekeley Prof. Dr. Ivo Lewin Küchler

Rio de Janeiro, dezembro de 2006 


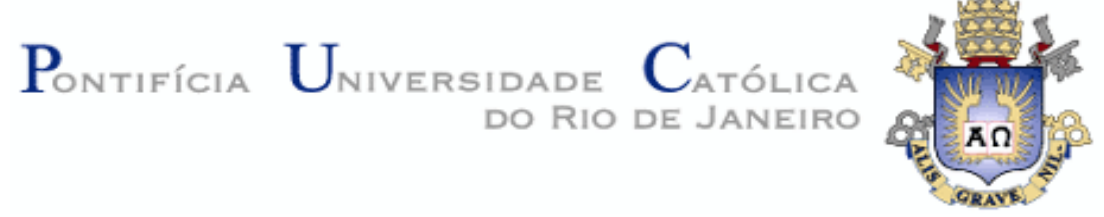

Roberta Amorim de Assis

\section{Aperfeiçoamento e aplicações de uma metodologia para análise de especiação de arsênio por eletroforese capilar com detector de ICPMS}

Tese apresentada como requisito parcial para obtenção do título de Doutor pelo Programa de Pós-Graduação em Química da PUC-Rio. Aprovada pela Comissão Examinadora abaixo assinada.

Prof. Dr. Norbert Miekeley

Orientador PUC-Rio

Prof. Dr. Ivo Lewin Küchler UFF

Profa. Dra. Cora Cunha Campos UFRJ

Dra. Shirley de Mello P. Abrantes FIOCRUZ

Prof. Dr. Ricardo Erthal Santelli UFF

Prof. Dr. Reinaldo Calixto de Campos

Prof. José Eugenio Leal Coordenador(a) Setorial do Centro Técnico Científico - PUC-Rio 
Todos os direitos reservados. É proibida a reprodução total ou parcial do trabalho sem autorização da universidade, da autora e do orientador.

Roberta Amorim de Assis

Graduou-se em Química Industrial pela Universidade Federal Fluminense, fazendo parte do quadro permanente de professores desta universidade desde 1990. Obteve seu título de Msc em Química Analítica pela Pontifícia Universidade Católica do Rio de Janeiro.

Ficha Catalográfica

Assis, Roberta Amorim de
Aperfeiçoamento e aplicações de uma
metodologia para análise de especiação de arsênio por
eletroforese capilar com detector de ICPMS / Roberta
Amorim de Assis ; orientador: Norbert Miekeley ; co-
orientador: Ivo Lewin Küchler . - 2006.
159 f. : il. ; 30 cm
Tese (Doutorado em Química)-Pontifícia
Universidade Católica do Rio de Janeiro, Rio de Janeiro,
2006. $\quad$ Inclui bibliografia
1. Química - Teses. 2. ICPMS. 3. Eletroforese
capilar. 4. Especiação de arsênio. 5. Contaminantes em
suco de uva. 6. Metabolismo de MMA (V) em cavalos. I.
Miekeley, Norbert. II. Küchler, Ivo Lewin. III Pontifícia
Universidade Católica do Rio de Janeiro. Departamento de
Química. IV. Título.

CDD: 540 
Ao Clauber e aos meus filhos, Michele e Vítor, pelo incentivo, apoio e compreensão. 


\section{Agradecimentos}

Aos meus orientadores, Norbert Miekeley e Ivo L. Küchler, pela orientação, confiança e apoio na realização deste trabalho.

À PUC-Rio pela bolsa recebida da VRAC.

Aos funcionários da PUC-Rio, em especial Adriana, Rodrigo, Anselmo e Noberto pela ajuda quando necessário.

Ao técnico Álvaro Jorge Pereira pelos ensinamentos sobre ICPMS, ajuda nas análises e amizade.

Aos estagiários do Laboratório de ICPMS da PUC-Rio, Paulo e Leonardo, pela ajuda nas análises.

Ao Dr. Joerg Feldman da Universidade de Aberdeen pela gentileza da doação dos padrões de As e Sb.

Aos colegas da PUC-Rio pelo apoio e incentivo.

Ao CENPES/Petrobrás pelo apoio financeiro e pelo empréstimo de um ICPMS, modelo Elan 6000.

À Burgener Research, AU pela doação do nebulizador Mira Mist CE.

Às minhas amigas Heloísa e Ana pelo apoio, incentivo e, principalmente, pela amizade que me fortaleceram nos momentos mais difíceis.

À UFF pela concessão do meu afastamento total.

Aos colegas do Departamento de Química Analítica, em especial minhas amigas Aída e Soly, pelo incentivo, apoio e pela amizade sempre presentes.

À Dra. Shirley de Mello P. Abrantes, do INCQS da FIOCRUZ, pela ajuda inicial 
no aprendizado sobre eletroforese capilar.

À diretora do Laboratório Antidoping do Jóquei Clube do Brasil, Marta Brandão Tozzi e seus colaboradores, pela importante cooperação no projeto sobre o metabolismo de MMA(V) em cavalos.

À minha mãe Lenira, meus irmãos Lúcia e Roberto, pelo carinho, incentivo e apoio emocional.

Aos integrantes da Comissão Examinadora. 


\section{Resumo}

Assis, Roberta Amorim, Miekeley, Norbert (orientador). Aperfeiçoamento e aplicações de uma metodologia para análise de especiação de arsênio por eletroforese capilar com detector de ICPMS. Rio de Janeiro, 2006. 159p. Tese de Doutorado - Departamento de Química, Pontifícia Universidade Católica do Rio de Janeiro.

A eletroforese capilar (CE) como técnica de separação está bem estabelecida para o uso em estudos de especiação elementar; porém, em muitas aplicações, a baixa sensibilidade devido à detecção UV, impede a obtenção de baixos limites de detecção (LD). Desta forma, tem sido de grande relevância o estudo do acoplamento (hifenação) entre a CE e sistemas de detecção mais sensíveis. Iniciou-se o trabalho com uma avaliação crítica entre diversas interfaces para o acoplamento entre a $\mathrm{CE}$ e a espectrometria de massas com plasma indutivamente acoplado (ICPMS). Foram testadas uma interface comercial e outras, adaptadas no próprio laboratório a partir de diferentes micronebulizadores e câmaras de nebulização. O estudo concluiu que o nebulizador de fluxo paralelo (Mira Mist CE, Burgener Research, AU) apresentou melhor performance, devido a ausência de efeito de retro-pressão acima do orifício do capilar de eletroforese, e por ter um canal de amostra suficientemente grande para introduzir o capilar até a saída do nebulizador, impedindo assim, o entupimento do mesmo em trabalhos de rotina, observado em todos os outros nebulizadores. Utilizando este nebulizador e uma câmara ciclônica de volume reduzido $(20 \mathrm{~mL})$, foi desenvolvida uma metodologia para separação eletroforética e quantificação por ICPMS de cinco espécies de arsênio: AsIII, AsV, MMAV, DMAV e AsB. Um estudo sistemático de diversos parâmetros experimentais resultou nas seguintes condições otimizadas para a separação e nebulização: tampão de fosfato $20 \mathrm{mmol}$ $\mathrm{L}^{-1}(\mathrm{pH}=9)$ com $1,5 \mathrm{mmol} \mathrm{L}^{-1}$ de TTAB como modificador do fluxo eletroosmótico; tempo de injeção no modo hidrodinâmico de 40 s a 50 mbar; e solução make up de $\mathrm{NH}_{4} \mathrm{NO}_{3} 20 \mathrm{mmol} \mathrm{L}^{-1}(\mathrm{pH}=9)$ com $10 \%$ de metanol, e vazão de $40 \mu \mathrm{L} \mathrm{min}{ }^{-1}$. Iodeto foi utilizado para monitorar a separação eletroforética, enquanto que $\mathrm{Cs}^{+}$foi usado para controlar a eficiência e constância de nebulização. Os desvios padrão relativos para a posição dos picos e as suas 
áreas correspondentes foram < $10 \%$ para todas as espécies, enquanto que os limites de detecção foram de $2,5 \mu \mathrm{g} \mathrm{L}^{-1}$ para $\mathrm{AsB}$ e de $0,5 \mu \mathrm{g} \mathrm{L}^{-1}$ para as demais. A metodologia foi aplicada para análise de especiação de arsênio em amostras de suco de uva, e num estudo preliminar sobre o metabolismo de Arsenil® (MMAV) em cavalos. No primeiro estudo, observou-se que as concentrações de As-total em todas as amostras analisadas (31 de 20 marcas de suco de uva diferentes), e também de outros elementos tóxicos (p.ex. $\mathrm{Al}, \mathrm{Hg}, \mathrm{Cd}, \mathrm{Sn}, \mathrm{Pb}, \mathrm{e} \mathrm{Al}$ ), encontravam-se abaixo dos "Limites Máximos de Tolerância" estabelecidos pela legislação brasileira. Estudos de especiação por CE-ICPMS e FIA-HG-ICPMS mostravam que entre os cinco espécies de arsênio estudadas, apenas as inorgânicas (AsV e AsIII) estavam presentes, em proporções variáveis. O estudo sobre a absorção e excreção da droga Arsenil® (MMAV) em cavalos mostrou que a droga é rapidamente eliminada pelo organismo, sugerindo uma cinética de primeira ordem para o processo, durante o tempo investigado (sete dias), com tempos de meia vida de aproximadamente $34 \mathrm{~h}$ (urina) e $44 \mathrm{~h}$ (plasma), respectivamente. Observou-se a presença de DMAV na urina como único metabólito da droga, já presente nos primeiros dois dias da aplicação, e acompanhando o perfil de absorção e excreção do Arsenil® (MMAV). Estes resultados indicam que a metilação é a via principal de desintoxificação, confirmando observações já feitas por diversos autores, mas em outros sistemas biológicos.

\section{Palavras-chave}

ICPMS; eletroforese capilar; especiação de arsênio; contaminantes em suco de uva; metabolismo de MMAV em cavalos. 


\section{Abstract}

Assis, Roberta Amorim, Miekeley, Norbert (orientador). Improvements and applications of a methodology for the speciation analysis of arsenic using capillary electrophoresis with ICPMS detector. Rio de Janeiro, 2006. 159p. Doctoral Thesis, Pontifícia Universidade Católica, Rio de Janeiro.

Capillary electrophoresis (CE) is a well established separation technique for the study of elemental speciation, however, its low electroosmotic flow ( $\mathrm{nL} \mathrm{min}^{-1}$ ) restricts its application in many studies in which low detection limits are required. For this reason, the study of $\mathrm{CE}$ coupling (hyphenation) to more sensitive detectors is of great relevance. This work was initiated with a critical evaluation of different interfaces of CE to ICPMS, including a commercial interface, and others, mounted in our laboratory from different micronebulizers and spray chambers. Best results in routine work were obtained with a parallel flow nebulizer (Mira Mist CE, Burgener Research, AU) due to the absence of backpressure on the capillary orifice and its large internal sample channel, which permits that the $\mathrm{CE}$ capillary can be inserted up to the nebulizer exit, thus avoiding clogging problems observed in all other nebulizers. Using this nebulizer in combination with a small-volume cyclonic spray chamber $(20 \mathrm{~mL})$, a method was developed for the electrophoretic separation and quantification by ICPMS of five arsenic species: AsIII, AsV, MMAV, DMAV and AsB. The systematic study of different experimental parameters resulted in the following optimized separation and nebulization conditions: phosphate buffer $(\mathrm{pH}=9.0), 20 \mathrm{mmol} \mathrm{L}^{-1}$ with $1.5 \mathrm{mmol} \mathrm{L}^{-1}$ TTAB as electroosmotic flow modifier; injection time (hydrodynamic mode) of $40 \mathrm{~s}$ at 50 mbar; make-up solution of $\mathrm{NH}_{4} \mathrm{NO}_{3} 20 \mathrm{mmol}$ $\mathrm{L}^{-1}(\mathrm{pH}=9)$ containing $10 \%$ of methanol and injected at flow of $40 \mu \mathrm{L} \mathrm{min}^{-1}$. Iodide was used as a monitor for the electrophoretic separation, whereas $\mathrm{Cs}^{+}$was applied for controlling the nebulization efficiency and constancy. Repeatabilities (RSD) in peak location and peak area measurements were better than $10 \%$ in all cases, and detection limits were about $2.5 \mu \mathrm{g} \mathrm{L}^{-1}$ for $\mathrm{AsB}$ and $0.5 \mu \mathrm{g} \mathrm{\textrm {L } ^ { - 1 }}$ for all other species studied. The methodology was applied in the speciation analysis of arsenic in grape juice samples, and in a preliminary study on the uptake, transformation and excretion of Arsenil ${ }^{\circledR}\left(\mathrm{MMA}^{\mathrm{V}}\right)$ by horses. In the first study, low concentrations o total arsenic and other toxic elements (e.g Al, $\mathrm{Hg}, \mathrm{Cd}, \mathrm{Sn}$, 
$\mathrm{Pb}$, e $\mathrm{Al}$ ) were measured in 31 samples (20 different brands) and which were in accordance with "Maximum Tolerance Levels" established by Brazilian laws. Speciation analysis of these samples by CE-ICPMS and FIA-HG-ICPMS revealed the existence, in varying proportions, of only inorganic arsenic species $\left(\mathrm{As}^{\mathrm{V}}\right.$ and $\left.A s^{I I I}\right)$. The investigations on the uptake and excretion of Arsenil ${ }^{\circledR}\left(\mathrm{MMA}^{\mathrm{V}}\right)$ by horses showed that the drug is rapidly eliminated from the organism, suggesting a first order kinetics for the excretion/elimination with half lives of about $34 \mathrm{~h}$ and $44 \mathrm{~h}$ for urine and plasma, respectively, within the time period of seven days here studied. $\mathrm{DMA}^{\mathrm{V}}$ was the only metabolic alteration product of Arsenil® detected already in the first two days after drug uptake and accompanying its absorption and excretion profile. These results indicate that methylation is the principal detoxification pathway, as already observed by other authors but for different biologic systems.

Key-words: ICPMS; capillary electrophoresis; arsenic speciation; contaminants in grape juice; metabolism of $\mathrm{MMA}^{\mathrm{V}}$ in horses. 


\section{Sumário}

1 Introdução e objetivos 23

1.1. Importância da especiação 23

1.2. Alguns aspectos do acoplamento entre eletroforese capilar e a espectrometria de massas com plasma indutivamente acoplado 25

1.3. Objetivos deste trabalho 26

2 Teoria Geral sobre Eletroforese Capilar 28

2.1. Breve resumo sobre a técnica de eletroforese capilar 28

2.1.1. Eletroforese capilar de zona (CZE) 28

2.1.2. Cromatografia eletrocinética micelar (MEKC) 29

2.1.3. Isotacoforese Capilar (CITP) 30

2.1.4. Focalização Isoelétrica Capilar (CIEF) 31

2.1.5. Eletroforese Capilar em Gel (CGE) 32

2.1.6. Eletrocromatografia Capilar (CEC) 33

2.2. Aspectos teóricos sobre a eletroforese capilar de zona 34

3 Revisão bibliográfica sobre o acoplamento entre eletroforese capilar e espectrometria de massas com plasma indutivamente acoplado (ICPMS)39

4 Arsênio: Breve resumo sobre a especiação deste elemento e de suas propriedades toxicológicas $\quad 50$

4.1. Fontes de arsênio $\quad 50$

4.2. Toxicologia do arsênio nos seres humanos 52

4.3. Toxicologia do arsênio nos animais 54

4.3.1. Determinação de arsênio como exame antidoping em cavalos de corrida

4.4. Espécies de arsênio no ambiente e estabilidade das soluções 57

4.5. Análise de especiação de arsênio 59

5 Materiais e Métodos 
5.1. Eletroforese capilar 62

5.1.1. Condicionamento dos capilares 63

5.2. Espectrômetro de massas com plasma indutivamente acoplado (ICPMS) 65

5.3. Interfaces utilizadas entre CE e ICPMS 68

5.4. Preparo de reagentes e padrões 69

5.5. Coleta e preparo das amostras $\quad 71$

5.5.1. Urina, sangue, plasma e pêlo 71

$\begin{array}{ll}\text { 5.5.2. Suco de uva } & 73\end{array}$

5.6. Análise por geração de hidretos $\quad 74$

6 Resultados e discussões $\quad 76$

6.1. Comparação crítica das interfaces CE-ICPMS 76

6.1.1. Interface CEI-100 77

6.1.2. O nebulizador MCN-100 84

6.1.3. Nebulizador MicroMist $\quad 87$

6.1.4. Nebulizador "Mira Mist CE" 91

6.2. Otimização e validação da metodologia para a especiação de arsênio 96

6.2.1. Otimização do pH do tampão de separação 96

6.2.2. Otimização da concentração do tampão de separação 100

6.2.3. Otimização da concentração do tensoativo TTAB 102

6.2.4. Otimização da voltagem 105

6.2.5. Parâmetros relacionados à introdução da amostra no ICPMS 108

6.3. Validação de metodologias analíticas

6.3.1. Separação e quantificação de espécies de arsênio por CE-ICPMS

6.3.2. Quantificação de arsênio por FIA-HG-ICPMS

6.3.3. Quantificação de As e outros elementos por ICPMS com calibração externa 
6.4.1.2. Especiação de arsênio em amostras de suco de uva

6.4.2. Análise do sangue, plasma, urina e pelo de crina em cavalos tratados com MMA (Arsenil $\circledast$ )

6.4.2.1. Concentração total de arsênio e de alguns outros elementos em amostras de crina de cavalo

6.4.2.2. Concentração de arsênio total em amostras de sangue, plasma e urina de cavalo antes, durante e depois do tratamento com Arsenil@

6.4.2.3. Análise de especiação de arsênio em amostras de urina

7 Conclusões e considerações finais 


\section{SIGLAS}

AFS - Espectrometria de fluorescência atômica

As(III) - Arsenito

As $(\mathrm{V})$ - Arsenato

AsB - Arsenobetaína

AsC - Arsenocolina

AAS - Espectrometria de absorção atômica

CZE - Eletroforese capilar de zona

CEC - Eletrocromatografia capilar

CGE - Eletroforese capilar em gel

CIEF - Focalização isoelétrica capilar

CITP - Isotacoforese capilar

DMA(III) - Ácido dimetil arsenoso

$\operatorname{DMA}(\mathrm{V})$ - Ácido dimetil arsínico

FEI - Federação eqüestre internacional

FIA - Análise por injeção em fluxo

FSC - Cromatografia de fluido supercrítico

GC - Cromatografia gasosa

HG - Geração de hidretos

HPLC - Cromatografia líquida de alta eficiência

ICPOES - Espectrometria de emissão óptica com plasma indutivamente acoplado

ICPMS - Espectrometria de massas com plasma indutivamente acoplado

IUPAC - International Union of Pure and Applied Chemistry

MEKC - Cromatografia eletrocinética micelar

MMA(III) - Ácido monometil arsenoso

MMA(V) - Ácido monometil arsínico

$\mu_{\text {ef }}$ - Mobilidade eletroforética

$\mu_{\text {feo }}-$ Mobilidade eletroosmótica

SEC - Cromatografia de exclusão por tamanho

TTAB - Brometo de tetradeciltrimetil amônio 


\section{Lista de figuras}

Figura 1: Princípio da eletroforese de zona em solução livre. (a) estado inicial, (b) zonas distintas de amostra, gerando uma migração diferencial.Adaptado de Kuhn \& Hoffstetter-Kuhn (1993)

Figura 2: Representação esquemática do princípio da cromatografia

eletrocinética micelar. Adaptada de Kuhn \& Hoffstetter-Kuhn (1993)

Figura 3: Princípio da isotacoforese (a) Estado inicial, (b) Estado intermediário,

(c) Estado em equilíbrio. Adaptado de Kuhn \& Hoffstetter-Kuhn (1993)

Figura 4: Princípio da focalização isoelétrica. a) geração do gradiente de $\mathrm{pH}$,

b) introdução da amostra, c) estado estacionário. Adaptado da referência:

Kuhn \& Hoffstetter-Kuhn, 1993

Figura 5: Princípio da Eletroforese Capilar em Gel. Adaptado da referência:

Baker, 1995

Figura 6:Esquemadeumsistema de eletroforese capilar

(http://www.ceandcec.com)

Figura 7: Representação esquemática da parede do capilar. feo =

fluxo eletroosmótico (http://www.ceandcec.com)

Figura 8: Representação esquemática da migração de cátions e ânions na presença do fluxo eletroosmótico. $\mu$ ep é a mobilidade eletroforética,EOF = fluxo eletromótico. (http://www.ceandcec.com)

Figura 9: Equipamento para eletroforese capilar utilizado neste trabalho.

Figura 10: Cassetes e capilares para acoplamento com ICPMS (a) e para detecção UV (b).

Figura 11: ICPMS Elan 6000 no Laboratório de Espectrometria de Massas da PUC-Rio.

Figura 12: Interface CEI-100 (Cetac Technology, EUA). (1) conector “em cruz" mostrando as entradas: (2) do capilar (CAP), (3) do contato elétrico (CON) e (4) do tubo para conduzir o líquido make up (MU).(5) nebulizador microconcêntrico (MCN) inserido na câmara de nebulização (6).

Figura 13: Variação da Taxa de aspiração em função da vazão do gás de nebulização. Solução de In $10 \mu \mathrm{g} \mathrm{L}^{-1}$. a) $1,0 \mathrm{~L} \mathrm{~min}^{-1}$, b) $1,1 \mathrm{~L} \mathrm{~min}^{-1}$, 
c) 1,2 $\mathrm{L} \mathrm{min}^{-1}$,d) 1,2 $\mathrm{L} \mathrm{min}{ }^{-1}$, e) $0,9 \mathrm{~L} \mathrm{~min}^{-1}$, f) 1,05 $\mathrm{L} \mathrm{min}^{-1}$.

Figura 14: Medição da estabilidade do sinal de In $10 \mu \mathrm{g} \mathrm{L}^{-1}$. Ar $=1,05 \mathrm{~L} \mathrm{~min}^{-1} .79$

Figura 15: Eletroferograma de uma solução de Rb $200 \mu \mathrm{g} \mathrm{L}^{-1} . \mathrm{V}=30 \mathrm{kV}$.

$\mathrm{I}=50 \mathrm{mbar} / 60 \mathrm{~s}$. Tampão: Borato $20 \mathrm{mmol} \mathrm{L}^{-1}, \mathrm{pH}=9,3$. Make up: Borato $10 \mathrm{mmol} \mathrm{L}^{-1}, \mathrm{pH}=9,3$.

Figura 16: Separação de Cs e Li por eletroforese capilar e ICPMS. Tampão:

Borato $20 \mathrm{mmol} \mathrm{L}{ }^{-1}, \mathrm{pH}=9,3 . \mathrm{V}=-30 \mathrm{kV} . \mathrm{I}=50 \mathrm{mbar} / 60 \mathrm{~s}$.

Figura 17: Eletroferograma de $\mathrm{Sb}(\mathrm{III}) 100 \mu \mathrm{g} \mathrm{L}^{-1} . \mathrm{I}=50 \mathrm{mbar} / 180 \mathrm{~s} . \mathrm{V}=-30$

kV. Tampão: Fosfato $10 \mathrm{mmol} \mathrm{L}^{-1}+$ TTAB $0,5 \mathrm{mmol} \mathrm{L}^{-1}, \mathrm{pH}=6,5$.

Make up: Fosfato $10 \mathrm{mmol} \mathrm{L}^{-1}, \mathrm{pH}=6,5$.

Figura 18: Eletroferograma de $\mathrm{Sb}(\mathrm{V}) 100 \mu \mathrm{g} \mathrm{L}^{-1} . \mathrm{I}=50 \mathrm{mbar} / 180 \mathrm{~s} . \mathrm{V}=-30$

kV. Tampão: Fosfato $10 \mathrm{mmol} \mathrm{L}^{-1}+$ TTAB 0,5 mmol L ${ }^{-1}, \mathrm{pH}=6,5$.

Make up: Fosfato $10 \mathrm{mmol} \mathrm{L}^{-1}, \mathrm{pH}=6,5$.

Figura 19: Separação de $\mathrm{Sb}(\mathrm{III})$ e $\mathrm{Sb}(\mathrm{V}) 100 \mu \mathrm{g} \mathrm{L}^{-1}$ cada. I = $50 \mathrm{mbar} / 5 \mathrm{~s}$. $\mathrm{V}=-30 \mathrm{KV}$. Tampão: Fosfato $10 \mathrm{mmol} \mathrm{L}^{-1}+\mathrm{TTAB} 0,5 \mathrm{mmol} \mathrm{L}^{-1}, \mathrm{pH}=6,5$. Make up: Fosfato $10 \mathrm{mmol} \mathrm{L}^{-1}, \mathrm{pH}=6,5$.

Figura 20: Separação de $\mathrm{Sb}(\mathrm{III}) 50 \mu \mathrm{g} \mathrm{L}^{-1}, \mathrm{Sb}(\mathrm{V})$ eTMSb $10 \mu \mathrm{g} \mathrm{L}^{-1}$ cada. $\mathrm{I}=50 \mathrm{mbar} / 5 \mathrm{~s} . \mathrm{V}=-30 \mathrm{KV}$. Tampão: Fosfato $10 \mathrm{mmol} \mathrm{L}^{-1}+$ TTAB 0,5 mmol L-1, $\mathrm{pH}=6,5$. Make up: Fosfato $10 \mathrm{mmol} \mathrm{L}^{-1}, \mathrm{pH}=6,5$.

Figura 21: Sinal de $\mathrm{Sb}(\mathrm{V}) 10 \mathrm{mg} \mathrm{L}^{-1}$. Vazão do gás de nebulização de 0,4 L. $\min ^{-1}$. Tampão: Fosfato $10 \mathrm{mmol} \mathrm{L}^{-1}+$ TTAB $0,5 \mathrm{mmol} \mathrm{L}^{-1}, \mathrm{pH}=9$. Make up: Fosfato $10 \mathrm{mmol} \mathrm{L}^{-1}+\mathrm{In} 10 \mu \mathrm{g} \mathrm{L}^{-1}, \mathrm{pH}=9 . \mathrm{I}=50 \mathrm{mbar} / 60 \mathrm{~s}$. $\mathrm{V}=-30 \mathrm{kV}$.

Figura 22: Sinal de $\mathrm{Sb}(\mathrm{V}) 10 \mathrm{mg} \mathrm{L}^{-1}$. Vazão do gás de nebulização de 0,5 L. $\min ^{-1}$. Tampão: Fosfato $10 \mathrm{mmol} \mathrm{L}^{-1}+$ TTAB $0,5 \mathrm{mmol} \mathrm{L}^{-1}, \mathrm{pH}=9$.

Make up: Fosfato $10 \mathrm{mmol} \mathrm{L}^{-1}+\mathrm{In} 10 \mu \mathrm{g} \mathrm{L}^{-1}, \mathrm{pH}=9 . \mathrm{I}=50 \mathrm{mbar} / 60 \mathrm{~s}$. $\mathrm{V}=-30 \mathrm{kV}$.

Figura 23: Sinal de $\mathrm{Sb}(\mathrm{V})$. Vazão do gás de nebulização de $0,6 \mathrm{~L} \mathrm{~min}^{-1}$. Tampão: Fosfato $10 \mathrm{mmol} \mathrm{L}^{-1}+$ TTAB 0,5 mmol L ${ }^{-1}, \mathrm{pH}=9$. Make up: Fosfato $10 \mathrm{mmol} \mathrm{L}^{-1}+\mathrm{In} 10 \mu \mathrm{g} \mathrm{L}^{-1}, \mathrm{pH}=9 . \mathrm{I}=50 \mathrm{mbar} / 60 \mathrm{~s} . \mathrm{V}=-30 \mathrm{kV}$.

Figura 24: Sinal de $\mathrm{Sb}(\mathrm{V})$. Vazão do gás de nebulização de $0,7 \mathrm{~L} \mathrm{~min}^{-1}$. Tampão: Fosfato $10 \mathrm{mmol} \mathrm{L}^{-1}+$ TTAB 0,5 mmol L ${ }^{-1}, \mathrm{pH}=9$. Make up: Fosfato $10 \mathrm{mmol} \mathrm{L}^{-1}+\mathrm{In} 10 \mu \mathrm{g} \mathrm{L}^{-1}, \mathrm{pH}=9 . \mathrm{I}=50 \mathrm{mbar} / 60 \mathrm{~s} . \mathrm{V}=-30 \mathrm{kV}$. 
Figura 25: Interface composta de uma peça em formado de "cruz" acoplado ao nebulizador MicroMist.

Figura 26: Separação de $\mathrm{Sb}(\mathrm{V})$ e $\mathrm{As}(\mathrm{V}) 100 \mu \mathrm{g} \mathrm{L} \mathrm{L}^{-1}$ cada. $\mathrm{I}=50 \mathrm{mbar} / 30 \mathrm{~s}$.

$\mathrm{V}=-30 \mathrm{KV}$. Tampão: Fosfato $20 \mathrm{mmol} \mathrm{L}^{-1}+\mathrm{TTAB} 0,5 \mathrm{mmol} \mathrm{L}^{-1}, \mathrm{pH}=9$.

Make up: $\mathrm{NH}_{4} \mathrm{NO}_{3} 20 \mathrm{mmol} \mathrm{L}{ }^{-1}+\mathrm{Cs} 0,5 \mu \mathrm{g} / \mathrm{L}, \mathrm{pH}=9$.

Figura 27: Eletroferograma do MMA $100 \mu \mathrm{g} \mathrm{L}^{-1} . \mathrm{I}=50 \mathrm{mbar} / 5 \mathrm{~s}$, $\mathrm{V}=-14 \mathrm{KV}$.Tampão: Fosfato $20 \mathrm{mmol} \mathrm{L}^{-1}+$ TTAB $0,5 \mathrm{mmol} \mathrm{L}{ }^{-1}, \mathrm{pH}=9$.

Make up: $\mathrm{NH}_{4} \mathrm{NO}_{3} 20 \mathrm{mmol} \mathrm{L}{ }^{-1}+\mathrm{Cs} 0,2 \mu \mathrm{g} \mathrm{L}-1, \mathrm{pH}=9$.

Figura 28: Eletroferograma do DMA $100 \mu \mathrm{g} \mathrm{L}^{-1} . \mathrm{I}=50 \mathrm{mbar} / 5 \mathrm{~s}$.

$\mathrm{V}=-14 \mathrm{KV}$. Tampão: Fosfato $20 \mathrm{mmol} \mathrm{L}{ }^{-1}+$ TTAB 0,5 mmol L ${ }^{-1}, \mathrm{pH}=9$.

Make up: $\mathrm{NH}_{4} \mathrm{NO}_{3} 20 \mathrm{mmol} \mathrm{L}{ }^{-1}+\mathrm{Cs} 0,2 \mu \mathrm{g} \mathrm{L}^{-1}, \mathrm{pH}=9$.

Figura 29: Eletroferograma do As(V) $100 \mu \mathrm{g} \mathrm{L}^{-1} . \mathrm{I}=50 \mathrm{mbar} / 5 \mathrm{~s}$.

$\mathrm{V}=-30 \mathrm{KV}$. Tampão: Fosfato $10 \mathrm{mmol} \mathrm{L}^{-1}+\mathrm{TTAB} 0,5 \mathrm{mmol} \mathrm{L}^{-1}, \mathrm{pH}=6,5$.

Make up: $\mathrm{NH}_{4} \mathrm{NO}_{3} 20 \mathrm{mmol} \mathrm{L}{ }^{-1}+\mathrm{Cs} 0,2 \mu \mathrm{g} \mathrm{L}^{-1} \mathrm{In}, \mathrm{pH}=9$.

Figura 30: Apresentação esquemática da construção e funcionamento do nebulizador Mira Mist CE com conector "T" para eletroforese capilar. F = flow spoiler. Esquema adaptado do folheto de informação da Burgener Research $(\mathrm{Au})$.

Figura 31: Foto apresentando o acoplamento CE-ICPMS usando como interface o nebulizador Mira Mist CE. No detalhe (a) mostra-se o sistema nebulizador/câmara de nebulização com o "T" e a conexãoelétrica e no (b) a conexão da interface com o ICPMS.

Figura 32: Eletroferograma do $\mathrm{Sb}(\mathrm{V}) 100 \mu \mathrm{g} \mathrm{L} \mathrm{L}^{-1} . \mathrm{I}=50 \mathrm{mbar} / 10 \mathrm{~s}$.

$\mathrm{V}=-20 \mathrm{KV}$. Tampão: Fosfato $20 \mathrm{mmol} \mathrm{L}^{-1}+\mathrm{TTAB} 0,5 \mathrm{mmol} \mathrm{L}^{-1}, \mathrm{pH}=9$.

Make up: $\mathrm{NH}_{4} \mathrm{NO}_{3} 20 \mathrm{mmol} \mathrm{L}{ }^{-1}+\mathrm{Cs} 0,2 \mu \mathrm{g} \mathrm{L}^{-1}, \mathrm{pH}=9$.

Figura 33: Aspiração de uma solução de Cs $1 \mu \mathrm{g} \mathrm{L}^{-1}$, utilizando-se vazões de aspiração diferentes (em $\mu \mathrm{L} \mathrm{min}^{-1}$ ) (a) 25; (b) 40 e (c) 60 .

Figura 34: Eletroferograma da separação de espécies de As em pH’s diferentes. 98 pH: (a) 7,5; (b) 8,0 e (c) 8,5 Picos: 1. As(V), 2. MMA(V), 3. DMA(V) + AsIII, 4. AsB.

Figura 35: Eletroferograma da separação de espécies de As em pH’s diferentes.

pH: (a) 9,0; (b) 9,5. Picos: 1. As(V), 2. MMA(V), 3. DMA(V) 4. AsIII,5. AsB. 99 Figura 36: Eletroferograma da separação de espécies de As em concentrações 
de tampão diferentes. Tampão: fosfato, contendo TTAB $0,5 \mathrm{mmol} \mathrm{L}^{-1}, \mathrm{pH}=9,0$

(a) $5 \mathrm{mmol} \mathrm{L}{ }^{-1}$; (b) $10 \mathrm{mmol} \mathrm{L}$. Picos: 1.As(V), 2.MMA(V), 3. DMA(V), 4. As(III). 5. AsB.

Figura 37: Eletroferograma da separação de espécies de As em concentrações de tampão diferentes. Tampão: fosfato, contendo TTAB $0,5 \mathrm{mmol} \mathrm{L}^{-1}, \mathrm{pH}=9,0$ (a) $15 \mathrm{mmol} \mathrm{L}{ }^{-1}$; (b) $20 \mathrm{mmol} \mathrm{L}{ }^{-1}$. Picos: 1.As(V), 2.MMA(V), 3. DMA(V), 4. As(III). 5. AsB.

Figura 38: Eletroferograma da separação de espécies de arsênio em concentrações de TTAB diferentes. (a) TTAB 0,3 mmol L ${ }^{-1}$; (b) TTAB 1,0 mmol L ${ }^{-1}$. Picos: 1.As(V), 2.MMA(V), 3. DMA(V), 4. As(III).

Figura 39: Eletroferograma da separação de espécies de arsênio em concentrações de TTAB diferentes: (a) TTAB 1,5 mmol L ${ }^{-1}$; (b) TTAB 2,0 mmol L-1. Picos: 1. As(V), 2. MMA(V), 3. DMA(V), 4. As(III).

Figura 40: Eletroferograma da separação de 5 espécies de arsênio. Tampão: Fosfato $20 \mathrm{mmol}^{-1}+$ TTAB $1,5 \mathrm{mmol} \mathrm{L}-1, \mathrm{pH}=9$. Make up: $\mathrm{NH}_{4} \mathrm{NO}_{3}$ $20 \mathrm{mmol} \mathrm{L} \mathrm{L}^{-1}+\mathrm{Cs} 1 \mu \mathrm{g} \mathrm{L}^{-1}+10 \%$ de metanol, $\mathrm{pH}=9$. Vazão da bomba: $20 \mu \mathrm{L} \mathrm{min}{ }^{-1}, \mathrm{Ar}=1,0 \mathrm{~L} \mathrm{~min}^{-1} . \mathrm{I}=50 \mathrm{mbar} / 40 \mathrm{~s} . \mathrm{V}=-28 \mathrm{kV} . \mathrm{T}=20^{\circ} \mathrm{C}$. Picos: 1. As(V), 2. MMA(V), 3. DMA(V), 4. As(III), 5. AsB.

Figura 41: Função característica de voltagem x corrente em diferentes temperaturas. Eletrólito: solução tampão de fosfato $20 \mathrm{mmo} \mathrm{L}^{-1}+$ TTAB 1,5 $\mathrm{mmol} \mathrm{L}^{-1}(\mathrm{pH}=9)$. Equipamento: Agilent HP-CE 3D.

Figura 42: Eletroferograma da separação de 5 espécies de arsênio.

Tampão: Fosfato $20 \mathrm{mmol} \mathrm{L}^{-1}+\mathrm{TTAB} 1,5 \mathrm{mmol} \mathrm{L}^{-1}, \mathrm{pH}=9$. Make up: $\mathrm{NH}_{4} \mathrm{NO}_{3} 20 \mathrm{mmol} \mathrm{L}{ }^{-1}+\mathrm{Cs} 1 \mu \mathrm{g} \mathrm{L}^{-1}+10 \%$ de metanol, $\mathrm{pH}=9 . \mathrm{I}=50 \mathrm{mbar} /$ $20 \mathrm{~s}, \mathrm{~V}=-25 \mathrm{kV}$ a $22{ }^{\circ} \mathrm{C}$ Pico de referência: $\mathrm{I}^{-} 50 \mu \mathrm{g} \mathrm{L}{ }^{-1}$. Picos: 1 . As(V), 2. MMA(V), 3. DMA(V), 4. As(III) $100 \mu \mathrm{g} \mathrm{L}^{-1}$ cada e 5. AsB $50 \mu \mathrm{g} \mathrm{L}^{-1}$.

Figura 43: Variação da intensidade dos sinais de As e Cs em função da vazão do gás de nebulização (argônio). Nebulizador Mira Mist CE com câmara ciclônica (mod. Cinnabar, $20 \mathrm{~mL}$ ) (a) As; (b) Cs.

Figura 44: Estudo da estabilidade do sinal de Cs $\left(1 \mu \mathrm{g} \mathrm{L}^{-1}\right)$ em função de vazões

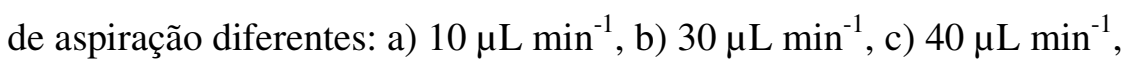
d) $50 \mu \mathrm{L} \mathrm{min}{ }^{-1}$. (Bomba HPLC mod. ABI 1400; nebulizador Mira Mist CE com câmara ciclônica). 
Figura 45: Separação de 5 espécies de arsênio. Condições experimentais:

Tampão: Fosfato $20 \mathrm{mmol} \mathrm{L}^{-1}+$ TTAB $1,5 \mathrm{mmol} \mathrm{L}^{-1}, \mathrm{pH}=9$. Make up:

$\mathrm{NH}_{4} \mathrm{NO}_{3} 20 \mathrm{mmol} \mathrm{L}{ }^{-1}+\mathrm{Cs} 1 \mu \mathrm{g} \mathrm{L}{ }^{-1}+10 \%$ metanol, $\mathrm{pH}=9 . \mathrm{I}=50 \mathrm{mbar} / 20 \mathrm{~s}$,

$\mathrm{V}=-25 \mathrm{kV}$ a $22^{\circ} \mathrm{C}$, Vazão da bomba: $40 \mu \mathrm{L} \min ^{-1}, \mathrm{Ar}=0,95 \mathrm{~L} \mathrm{~min}^{-1}$.

Pico de referência: I': $50 \mu \mathrm{g} \mathrm{L}^{-1}$; Picos: 1. As(V), 2. MMA(V), 3. DMA(V),

4. As(III) $100 \mu \mathrm{g} \mathrm{L}^{-1}$ cada; 5. AsB: $50 \mu \mathrm{g} \mathrm{L}^{-1}$.

Figura 46:Curvas analíticas de cinco espécies de arsênio determinados por

CE-ICPMS [1: As(III), $\mathrm{R}^{2}=0,992 ; 2:$ DMA, $\mathrm{R}^{2}=0,997 ; 3: \mathrm{MMA}^{2}=$

0,999; 4: $\left.\mathrm{As}(\mathrm{V}), \mathrm{R}^{2}=0,996 ; 5: \mathrm{AsB}, \mathrm{R}^{2}=0,993\right]$.

Figura 47: Visualização dos picos das espécies de arsênio na região limite.

A) padrão de $2,5 \mu \mathrm{g} \mathrm{L}^{-1}$ cada e b) padrão de $0,5 \mu \mathrm{g} \mathrm{L}^{-1}$ cada. Picos: 1 . As(V),

2. MMA(V), 3. DMA(V) e 4.As(III).

Figura 48: a) Exemplos Sinais transientes para As produzidos pelo sistema

FIAS 200 acoplado ao ELAN 5000 ICPMS; b) Chemifold com separador de membrana utilizado neste trabalho.

Figura 49: Curva de calibração típica para As obtida com o sistema FIAS 200 acoplado ao ELAN 5000 ICPMS. Alça de amostragem: $250 \mu \mathrm{L}$.

Figura 50: Exemplos de curvas de calibração para determinação quantitativa multielementar por ICPMS. Calibração externa com padronização dos sinais pelo ródio $(\mathrm{Rh})$.

Figura 51: Especiação de arsênio por CE-ICPMS em uma amostra de suco de uva (Dom Cândido) com concentração total de As de cerca de $20 \mu \mathrm{g} \mathrm{L}^{-1}$. Injeção: $50 \mathrm{mbar} / 40 \mathrm{~s}, \mathrm{~V}=-25 \mathrm{kV}, \mathrm{Ar}=0,96 \mathrm{~L} \mathrm{~min}^{-1}$.

Figura 52: Identificação dos picos em suco de uva (vide Figura 51) pela adição de $20 \mu \mathrm{g} \mathrm{L}^{-1}$ de $\mathrm{As}(\mathrm{V})$ e As(III), respectivamente.

Figura 53: Teores de arsênio em amostras de pêlo de crina dos três cavalos antes e depois do tratamento com Arsenil®.

Figura 54:Cinética de absorção e excreção da droga Arsenil@ monitorada através de amostras de sangue (total), plasma e urina do cavalo Cath Talescoean (Cta).

Figura 55:Cinética de absorção e excreção da droga Arsenil® monitorada através de amostras de sangue (total) e plasma em cavalos (Chaca eBlack Bits).137 Figura 56:Cinéticas de excreção de Arsenil ${ }^{\circledR}\left(\mathrm{MMA}^{\mathrm{V}}\right)$ medidas através da 
determinação de As em amostras de plasma e urina do cavaloCath Talescoean para o intervalo de tempo estudado, os resultados sugerem uma cinética de primeira ordem.

Figura 57: Eletroferograma do Arsenil® $\left(1000 \mu \mathrm{g} \mathrm{L}^{-1}\right)$. Tampão: fosfato $20 \mathrm{mmol} \mathrm{L}{ }^{-1}+$ TTAB $1,5 \mathrm{mmol} \mathrm{L}{ }^{-1}, \mathrm{pH}=9$. Make up: $\mathrm{NH}_{4} \mathrm{NO}_{3} 20 \mathrm{mmol} \mathrm{L}^{-1}$ $+\mathrm{Cs} \mu \mathrm{g} \mathrm{L} \mathrm{L}^{-1}+10 \%$ metanol. $\mathrm{I}=50 \mathrm{mbar} / 5 \mathrm{~s} . \mathrm{V}=-25 \mathrm{kV} \cdot \mathrm{Ar}=0,88 \mathrm{~L} \mathrm{~min}^{-1}$. Vazão do make up: $40 \mu \mathrm{L} \min ^{-1}$.

Figura 58: Eletroferograma obtido por CE-ICPMS da urina (1:5) do cavalo Cta no $5^{\circ}$ dia da administração do medicamento Arsenil®. Tampão: fosfato $20 \mathrm{mmol} \mathrm{L}{ }^{-1}+$ TTAB 1,5 mmol L-1 $\mathrm{pH}=9$. Make up: $\mathrm{NH}_{4} \mathrm{NO}_{3} 20 \mathrm{mmol} \mathrm{L}^{-1}$ $+\mathrm{Cs} \mu \mathrm{g} \mathrm{L} \mathrm{L}^{-1}+10 \%$ methanol. I $=50 \mathrm{mbar} / 5 \mathrm{~s} . \mathrm{V}=-25 \mathrm{kV} . \mathrm{Ar}=0,88$ L $\min ^{-1}$. Vazão do make up: $40 \mu \mathrm{L} \mathrm{min}{ }^{-1}$.

Figura 59: Eletroferograma mostrando a identificação do pico DMA(V) através da adição de um spike $\left(50 \mu \mathrm{g} \mathrm{L}^{-1}\right)$ na urina do cavalo Cta no $5^{\circ}$ dia da administração do medicamento Arsenil®. Condições experimentais semelhantes à Figura anterior.

Figura 60: Eletroferograma obtido imediatamente após a fortificação da urina com $500 \mu \mathrm{g} \mathrm{L}^{-1}$ de Arsenil®, sete dias e vinte e dois dias depois. Urina do cavalo Cta; condições experimentais semelhantes às da Figura 58.

Figura 61:Curva de excreção de MMA(V), DMA(V) e As-total na urina do cavalo CTA. Concentrações em $\mu \mathrm{g} \mathrm{L^{-1 }}$. 


\section{Lista de tabelas}

Tabela 1: Algumas interfaces entre CE e ICPMS utilizadas por diferentes autores na análise de especiação de diferentes elementos.

Tabela 2. Principais espécies de arsênio encontradas no meio ambiente.

(Adaptada de Tonietto, 2005).

Tabela 3 - Parâmetros operacionais/instrumentais utilizados em medidas de ICPMS.

Tabela 4: Diferentes sistemas de introdução de amostras testados neste trabalho.68

Tabela 5: Nome e código dos cavalos que participaram do estudo. 72

Tabela 6: Sucos de uva analisados para determinação de arsênio.

Tabela 7: Tempo de migração e seu desvio padrão relativo (DPR) para

diferentes espécies de As separadas e determinadas pela metodologia proposta $(\mathrm{n}=8)$.

Tabela 8: Comparação entre os desvios padrão relativos (DPR) na quantificação dos picos eletroforéticos por dois métodos diferentes $(\mathrm{n}=8)$.

Tabela 9: Concentração média de arsênio $\left(e m \mu \mathrm{g} \mathrm{L}^{-1}\right)$ determinada em três materiais de referência por FIA-HG-ICPMS.

Tabela 10: Coeficientes de determinação das curvas analíticas obtidas neste trabalho por ICPMS e limites de quantificação expressa como concentração equivalente ao branco (BEC) para suco de uva diluído 1:10.

Tabela 11: Resultados obtidos na análise quantitativa de dois materiais de referência (urina liofilizada) no Laboratório da PUC-Rio $(n=3 ; p=0.05)$.

Todas as concentrações em $\mu \mathrm{g} \mathrm{L}^{-1}$.

Tabela 12: Determinação de contaminantes inorgânicos em suco de uva. (ND: não determinado; * $\mathrm{Zn}, \mathrm{Na}$ e $\mathrm{Cu}$ em mg L ${ }^{-1}$, restante em $\mu \mathrm{g} \mathrm{L}{ }^{-1}$. $\mathrm{Na}$ e $\mathrm{Cr}$ foram determinados por ICP OES).

Tabela 13: Limites Máximos de Tolerância em suco de frutas. (Fontes: Coluna 2 -ANVISA Dec. $n^{\circ}$ 55.871/1965; Coluna 3 - Commision Regulation $n^{\circ}$ 466/2001. Off. J. Europ. Communities 77:9, 2001; coluna 4 - Indian PFA Act, 1954).

Tabela 14: Determinação de As-total, As(III) e As(V) em suco de uva. Todas 
as concentrações de em $\mu \mathrm{g} \mathrm{L}^{-1}$. (*) determinado após oxidação prévia da amostra (valores da Tabela 12).

Tabela 15: Amostras de suco de uva e seus respectivos conservantes (INS202: sorbato de potássio; INS211: benzoato de sódio; INS220: dioxido de enxofre; INS223: Metabissulfito de sódio).

Tabela 16:Concentrações de arsênio em amostras de pêlo de crina de cavalo antes (18/07/2006) e depois do tratamento (14/11/2006). Concentração de As em $\mu \mathrm{g} \mathrm{g}^{-1}$; a distância (em $\mathrm{cm}$ ) se refere ao segmento da amostra recolhida a partir do couro cabeludo.

Tabela 17: Composição inorgânica (mineralograma) de uma amostra de pêlo de crina do cavalo Chaca (Jóquei Clube do Brasil, Rio de Janeiro), mostrando baixas concentrações de elementos tóxicos quando comparadas com cabelo humano (vide Tabela 17).

Tabela 18: Dados de referência para diferentes elementos em amostras de cabelo de uma população de 1082 indivíduos (homens e mulheres com idade entre 25 e 55 anos) utilizando-se o critério $1 \sigma$, ou seja, $68 \%$ de uma distribuição log-normal). Para elementos tóxicos, o valor corresponde a $(+1 \sigma)$, para elementos "nutrientes" (essenciais), o intervalo é de $( \pm 1 \sigma)$, vide Carneiro et al., 2002.

Tabela 19: Concentrações médias de arsênio e outros parâmetros estatísticos para amostras de sangue e plasma dos cavalos que participaram neste estudo.

Concentração em $\mu \mathrm{g} \mathrm{L}^{-1}$.

Tabela 20: Concentração (em $\mu \mathrm{g} \mathrm{L}^{-1}$ ) das espécies MMA(V) e DMA(V) em amostras de urina do cavalo Cta.

Tabela 21: Comparação dos resultados de As em urina obtidos por dois métodos independentes (CE-ICPMS e FIA-HG-ICPMS). ND = não determinado. 